\title{
Covid-19 e tabagismo: relato de experiência sobre notificação de casos no Estado do Paraná
}

\author{
Covid-19 and smoking: experience report on case \\ notification in the State of Paraná
}

\begin{abstract}
Rejane Cristina Teixeira Tabuti ${ }^{1}$, Elaine Cristina Vieira de Oliveira ${ }^{2}$, Aureni de Souza Desplanches ${ }^{3}$

1. ORCID: https://orcid.org/0000-0002-0003-1793. Mestre em Psicologia Social Comunitária. Secretaria de Estado da Saúde, Curitiba, Paraná, Brasil. E-mail: rejane.teixeira@yahoo.com.br.

2. ORCID: https://orcid.org/0000-0002-8484-5259. Mestre em Alimentação e Nutrição. Secretaria de Estado da Saúde, Curitiba, Paraná, Brasil. E-mail: elainevolive@gmail.com.

3. ORCID: https://orcid.org/0000-0002-1065-6076. Especialista em Análise de Situação de Saúde e Gestão de Prevenção e Controle de Infecção Hospitalar. Secretaria de Estado da Saúde, Curitiba, Paraná, Brasil. E-mail: aurenidesplanches.sesa@gmail.com.
\end{abstract}

CONTATO: Rejane Cristina Teixeira Tabuti | Rua Piquiri, 170. Rebouças | rejaneteixeira@sesa. pr.gov.br | rejane.teixeira@yahoo.com.br

RESUMO $O$ tabagismo é a maior causa evitável isolada de adoecimento e morte precoce no Brasil e no mundo e tem sido relacionado à maior gravidade e risco de óbito por Covid-19. O Paraná foi o único Estado brasileiro a incluir o tabagismo como comorbidade na notificação de casos, experiência apresentada neste relato, juntamente a dados preliminares dos casos notificados no período de 1 de março a 30 de julho de 2020. No Paraná o tabagismo foi a segunda comorbidade mais frequente, entre os casos suspeitos; e a quarta entre os casos confirmados da doença, com baixa mortalidade. Os resultados preliminares agregam conhecimento ao adoecimento por Covid-19, contri- 
buem para a adoção de medidas com vistas a mitigar seu impacto no sistema de saúde e adoção de estratégias e políticas públicas para o enfrentamento deste importante problema de saúde pública.

DESCRITORES: Tabagismo. Infecções por coronavírus. Pandemias. Comorbidade.

\begin{abstract}
Smoking is the single largest preventable cause of illness and early death in Brazil and worldwide and has been linked to greater severity and risk of death from Covid-19. Paraná was the only Brazilian State to include smoking as a comorbidity in the notification of cases, an experience presented in this report, together with preliminary data from the cases notified from March 1 to July 30, 2020. In the state, smoking was the second most frequent comorbidity, among suspected cases; and the fourth among confirmed cases of the disease, with low mortality. The preliminary results add knowledge to the illness caused by Covid-19, contribute to the adoption of measures to mitigate its impact on the health system and the adoption of public strategies and policies to face this important public health problem.
\end{abstract}

DESCRIPTORS: Smoking. Coronavirus Infections. Pandemics. Comorbidity.

\title{
INTRODUÇÃO
}

A humanidade enfrenta uma das maiores crises decorrente da doença causada pelo vírus SARS-CoV-2 (Covid-19), declarada como Emergência de Saúde Pública de Importância Internacional (ESPII) em 11 de março de 2020. Pesquisadores relataram que o vírus foi detectado com maior frequência em amostras respiratórias na China, cuja transmissão ocorre por vias respiratórias e extrarrespiratórias, o que pode explicar a rápida disseminação da doença'.

Neste cenário, tem-se observado que o consumo de tabaco e seus derivados é um fator de risco para agravamento da Covid-192, além de ser considerado a maior causa evitável isolada de adoecimento e mortes precoces no Brasil, e em todo o mundo, comprometendo a capacidade pulmonar e causando variados tipos de inflamação. Substâncias tóxicas do cigarro enfraquecem o sistema 
imunológico, tornando os fumantes mais vulneráveis às infecções. As doenças crônicas não transmissíveis (DCNT) associadas ao tabagismo, como cardiovasculares, enfisema, câncer e diabetes, oferecem maior risco para complicações da Covid-193.

Fumantes parecem ser mais vulneráveis à infecção pelo coronavírus, pois o ato de fumar proporciona constante contato dos dedos (e possivelmente de cigarros contaminados) com os lábios, aumentando a possibilidade da transmissão do vírus para a boca. O uso de produtos que envolvem compartilhamento de bocais para inalar a fumaça - como narguilé (cachimbo d'água) e dispositivos eletrônicos para fumar (cigarros eletrônicos e cigarros de tabaco aquecido) - também podem facilitar a transmissão do vírus entre usuários e para a comunidade. Por isso, é possível dizer que o tabagismo é um agravante para a Covid-19, devido ao possível comprometimento da capacidade pulmonar, resultando em maiores chances de o fumante desenvolver sintomas graves da doença ${ }^{4}$.

Frente aos casos de Covid-19 no mundo, o Ministério da Saúde (MS) estabeleceu, em janeiro de 2020, a notificação compulsória e de forma imediata (até 24 horas) de casos suspeitos, prováveis e confirmados de infecção pelo coronavírus, devendo ser realizada pelo profissional de saúde responsável pelo atendimento ${ }^{5}$. As informações que constam nas notificações e aquelas registradas em prontuário fornecem os elementos para a longitudinalidade e coordenação do cuidado e que possibilitam a formulação de políticas e estratégias de saúde ${ }^{6}$.

Embora o tabagismo esteja nos documentos oficiais como condição de risco a ser considerado para possível complicação de síndrome gripal, este não se encontra na relação de comorbidades contidas na Ficha de Investigação de Síndrome Gripal (SG) de casos suspeitos de doença pelo coronavírus 2019 - Covid-19 (B34.2) disponibilizada pelo MS7.

Desde o Redcap, que foi inicialmente o sistema oficial de notificação de casos suspeitos de coronavírus e, posteriormente, o E-SUS-VE, verifica-se que o taba- 
gismo não esteve incluído como comorbidade na Ficha de Notificação de casos suspeitos de Covid-19.

O Estado do Paraná, atento ao tabagismo como condição de risco, incluiu este agravo no campo de comorbidades do sistema de notificação, desenvolvido pela Secretaria de Estado da Saúde do Paraná (SESA), para notificação de casos suspeitos de infecção pelo vírus SARS-CoV-2, denominado "Notifica Covid-19".

Portanto, o presente estudo tem por objetivo relatar a experiência do Paraná na inserção do tabagismo como comorbidade no sistema "Notifica Covid-19" e realizar análise descritiva dos casos notificados com esse campo preenchido.

\section{MÉTODO}

Trata-se de um relato de experiência que traz as etapas do processo que resultou na inserção do tabagismo como comorbidade no sistema de notificação de casos de Covid-19 no Paraná ("Notifica Covid-19"), contextualizada na perspectiva do trabalho das equipes técnicas participantes nesse processo.

Os dados descritos referem-se ao período de 1 de março a 30 de julho de 2020 e foram extraídos do sistema "Notifica Covid-19" e dos Boletins Epidemiológicos publicados pela SESA-PR. As informações são apresentadas segundo casos notificados, suspeitos e confirmados, sexo e idade, indicador mortalidade, e sua contextualização segundo a comorbidade tabagismo.

Conforme estabelecido no Parágrafo $1^{\circ}$ da Resolução 510 de 7 de abril de 2016, relativa à pesquisa envolvendo seres humanos, este relato utiliza informações agregadas, mantendo-se a confidencialidade dos casos e observando-se demais preceitos de ética em pesquisa, sendo dispensada apreciação por Comitê de Ética. 


\section{RESULTADOS E DISCUSSÃO}

No início de março, com a disponibilização pelo MS do formulário eletrônico Redcap para notificação de casos suspeitos de infecção pelo SARS-CoV-2, foi observada a ausência do tabagismo como campo para preenchimento no item de comorbidades. Fato este que causou preocupação na equipe da Divisão de Prevenção e Controle de Doenças Crônicas e Tabagismo da SESA, diante das evidências da doença como fator de agravamento para Covid-19.

As equipes do Centro de Informações Estratégicas em Vigilância em Saúde (CIEVS), da Coordenadoria de Vigilância Epidemiológica e do Núcleo de Informática e Informações, na busca por uma ferramenta que assegurasse informações relevantes diante da nova doença, buscou o desenvolvimento de um sistema próprio de notificação. Identificou-se, também, a necessidade de integração dos vários sistemas que traziam informações isoladas, a saber: E-SUS VE, Sistema de Informação de Vigilância da Gripe (SIVEP GRIPE) e Gerenciador de Ambiente Laboratorial (GAL).

A equipe da área técnica do tabagismo realizou articulação com as áreas responsáveis pelo desenvolvimento do novo sistema, no período de 26 a 31 de março, para a inclusão do tabagismo como comorbidade, elencando as suas formas, como cigarro convencional, eletrônico, com aditivos, narguilé, cachimbo, charuto e outros. Entretanto, diante da numerosa quantidade de informações já constantes, optou-se por inserir somente o campo tabagismo (Figura 1). Sua inclusão possibilitou a geração de informações relevantes para análise dos dados epidemiológicos por meio de dashboards que propiciam acompanhamento dos casos em tempo real. 
Figura 1. Ficha de Notificação de casos suspeitos de Covid-19 - “Notifica Covid-19” (1 ' versão).
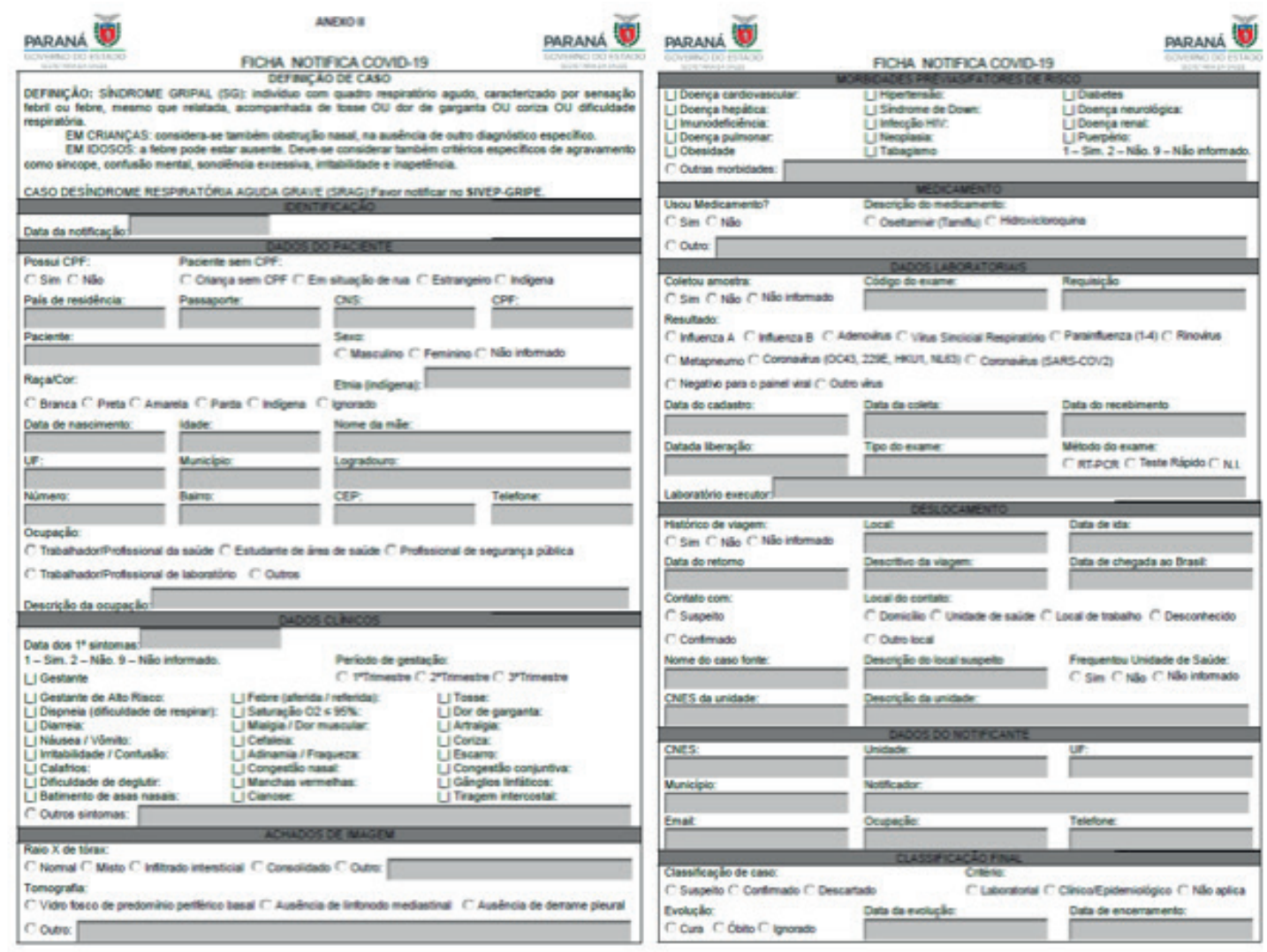

Fonte: SESA, 2020.

Na presente experiência, a aproximação e integração entre atenção e vigilância em saúde foram essenciais. Durante a pandemia, a articulação entre as áreas técnicas e demais serviços tem sido observada em todos os níveis de atenção, possibilitando a análise das condições e perfis de morbimortalidade, e trazendo elementos importantes para o planejamento das ações considerando as especificidades locais:

Em relação aos dados gerados pelo sistema, até o dia 30 de julho às 06:07 horas foram notificados 457.351 casos, sendo que as cinco comorbidades mais frequentes foram: Hipertensão (23,37\%), Tabagismo (14,86\%), Doença Pulmonar (11,15\%), Diabetes (7,98\%) e Doença Cardiovascular (7,95\%).

Após a investigação desses casos, foram confirmados 56.986, nos quais as cinco comorbidades mais frequentes estão apresentadas na Tabela 1. 
Tabela 1. Distribuição de casos confirmados de Covid-19 associados a comorbidades. Paraná, 2020.

\begin{tabular}{lcc}
\hline Comorbidade & N & \% \\
\hline Hipertensão & 2750 & 24,89 \\
Doença Cardiovascular & 1088 & 9,85 \\
Diabetes & 989 & 8,95 \\
Tabagismo & 967 & 8,95 \\
Doença Pulmonar & 834 & 7,55 \\
\hline
\end{tabular}

Fonte: “Notifica Covid-19”, 01/03/2020 a 30/07/2020, às 06:07 horas, SESA. Nota: *Dados sujeitos a alterações.

Dentre os 967 casos confirmados que apresentaram o tabagismo como comorbidade, $62,6 \%$ foram do sexo masculino e $37,2 \%$ do sexo feminino. Observa-se o expressivo número de casos confirmados que apresentam o tabagismo como uma das comorbidades na faixa etária de 20 a 29 anos. Identificou-se a ocorrência de tabagismo em < de 1 ano, o que representa uma inconsistência dos dados. $\mathrm{O}$ Gráfico 1 apresenta a distribuição dos casos confirmados segundo faixa etária.

Gráfico 1. Distribuição dos casos confirmados de Covid-19, em pacientes tabagistas, segundo a faixa etária. Paraná, 2020*.

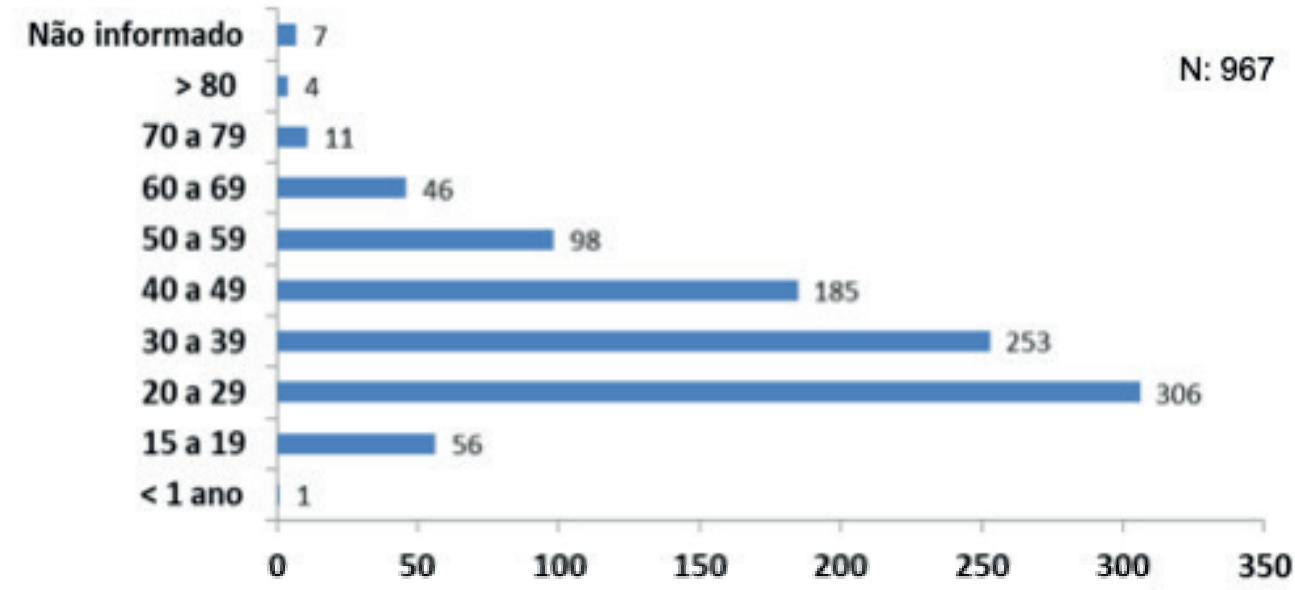

Nota: *Dados sujeitos a alterações.

O Paraná possui 399 municípios, os quais estão distribuídos no âmbito das 22 Regionais de Saúde (RS). Um total de 187 municípios apresentaram ao menos um 
caso confirmado com o campo tabagismo preenchido, sendo que em uma das RS não houve nenhum registro. Ainda $63,5 \%$ desses casos estavam concentrados em $5 \mathrm{RS}$, demonstrando que muitos serviços ignoraram esse campo no momento da notificação dos casos.

Com relação aos óbitos, foram notificados 1.792 até a data de 29 de julho, segundo o Boletim Epidemiológico ${ }^{9}$. Desse total, 1.045 foram qualificados e estão descritos conforme o tipo de comorbidade associada (Tabela 2), sendo 18 casos entre tabagistas. Destaca-se que as comorbidades mais frequentes nos óbitos investigados tem relação com o tabagismo, como é o caso das doenças cardiovasculares, pulmonares e câncer.

Tabela 2. Distribuição dos óbitos por Covid-19 qualificados segundo comorbidades associadas. Paraná, 2020a,b.

\begin{tabular}{lcc}
\hline COMORBIDADE & N & $\%$ \\
\hline Hipertensão Arterial Sistêmica & 258 & 23,9 \\
Diabetes Mellitus & 191 & 17,7 \\
Cardiopatia & 146 & 13,5 \\
Doença Pulmonar Crônica & 81 & 7,5 \\
Doença Renal Crônica & 69 & 6,4 \\
Acidente Vascular Cerebral & 68 & 6,3 \\
Neoplasias & 66 & 6,1 \\
Obesidade & 58 & 5,4 \\
Demência & 39 & 3,6 \\
Tabagismo & 18 & 1,7 \\
Trombose & 17 & 1,6 \\
Doença Hepática & 16 & 1,5 \\
Infarto do Miocárdio & 15 & 1,4 \\
Doença Neurológica & 13 & 1,2 \\
Hipotireoidismo & 10 & 0,9 \\
Doença Reumatológica & 7 & 0,6 \\
Doença Psiquiátrica & 3 & 0,3 \\
Etilismo & 3 & 0,3 \\
Outros & 2 & 0,2
\end{tabular}

Fonte: Boletim SESA, atualizado em 29/07/20209.

Nota: a) Dados sujeitos a alterações.

b) O número de óbitos constantes nos Sistemas CIEVS e Sistema de Informações sobre Mortalidade (SIM) é de 1.045, sendo que um mesmo caso pode ter mais de uma comorbidade. 
A Covid-19 pode ter implicações para os fumantes uma vez que o vírus afeta principalmente o sistema respiratório, causando frequentemente danos respiratórios leves a graves e que podem resultar em fatalidade. No entanto, como a Covid-19 é uma doença recentemente identificada, o vínculo entre o uso do tabaco e a doença precisa ser mais pesquisado e registrado. Há risco aumentado de sintomas mais graves e morte entre pacientes com Covid-19 que têm condições subjacentes, incluindo doenças cardiovasculares. Essa relação entre Covid-19 e saúde cardiovascular é importante porque o uso de tabaco e a exposição ao fumo passivo são as principais causas de doenças cardiovasculares, globalmente. Um sistema cardiovascular mais fraco entre os pacientes com Covid-19, com histórico de uso de tabaco, pode torná-los mais vulneráveis a sintomas graves, aumentando assim o risco para esses pacientes ${ }^{10}$.

Estudos têm relacionado a infecção por SARS-CoV-2 com o tabagismo e observado que fumantes ou pessoas com história de tabagismo apresentam maior risco para piores desfechos, como a morte $\mathrm{e}^{(2,11,12,13,14)}$. Em uma metanálise realizada com 19 estudos, os pesquisadores encontraram uma chance de cerca do dobro dos pacientes com história de tabagismo (fumante ou ex-fumante) evoluir para um desfecho mais desfavorável quando comparada aos não fumantes ${ }^{2}$. Em outra revisão sistemática, com metanálise, foi avaliada a relação entre fumar e Doença Pulmonar Obstrutiva Crônica (DPOC) e maior gravidade e/ou pior desfecho da Covid-19. O resultado apontou que fumantes tinham quase o dobro de chance de apresentar pior prognóstico quando comparados aos não fumantes, sendo que em pacientes com DPOC essa relação foi ainda mais forte ${ }^{13}$.

Esses resultados reforçam a necessidade de que mais estudos avaliem o perfil dos indivíduos infectados pelo SARS-CoV-2, especialmente em relação à existência de comorbidades, como o tabagismo, e sua associação com o risco para maior gravidade da Covid-19. Além da necessidade de melhor esclarecer os possíveis mecanismos da relação do hábito de fumar com piores desfechos para Covid-19. 
Este trabalho relata uma experiência bem sucedida de articulação na gestão, a qual resultou na inserção do campo tabagismo no sistema de notificação de casos de Covid-19, o que possibilita a obtenção de dados dos tabagistas infectados pelo SARS-CoV-2. Os dados apresentados nesse estudo demonstram o quanto essa experiência foi relevante, possibilitando análises sobre a contribuição do tabagismo para a ocorrência e/ou agravamento dos casos da doença.

É relevante apontar a potencial limitação existente, pois no momento da notificação a variável tabagismo pode não ter sido investigada. Outra limitação é que o sistema não possui campo para informar a condição de história de tabagismo (ex-fumantes), informação esta que poderia subsidiar análises importantes da relação do tabagismo com a Covid-19.

\section{CONCLUSÃO}

A inserção do tabagismo no "Notifica Covid-19" só foi possível pela integração e articulação entre atenção e vigilância em saúde. Os achados, embora sejam ainda preliminares, demonstram a presença do tabagismo de maneira importante tanto nos casos notificados quanto nos confirmados.

Além de ser um fator de risco para uma série de doenças crônicas, o tabagismo, assim como a Covid-19, se constitui em uma pandemia que atinge milhares de pessoas no mundo.

O levantamento dessas informações possibilita uma intervenção no sentido de reforçar a orientação aos profissionais de saúde sobre a importância do preenchimento qualificado de todos os campos da ficha de notificação, bem como da investigação dos casos para o seu encerramento, para que assim seja possível realizar análises mais robustas.

Estudos que avaliem a relação do tabagismo com infecções respiratórias, como é o caso da Covid-19, são necessários, uma vez que são doenças que têm causado grande impacto para a saúde pública. Para tanto, se fazem necessárias 
informações que possibilitem essas análises, sendo os sistemas de notificação um potencial instrumento para permitir a coleta e análise desses dados.

O relato de experiência é oportuno na medida em que apresenta uma experiência de sucesso, a partir da articulação de áreas da gestão em saúde, possibilitando a obtenção de informações estratégicas para a realização de diagnóstico situacional, o que é fundamental na criação, implementação, fortalecimento e reestruturação de políticas públicas.

\title{
AGRADECIMENTOS
}

\author{
Agradecemos à Profa. Dra. Elaine Drehmer de Almeida Cruz pelo incentivo e \\ contribuições ao texto.
}

\section{REFERÊNCIAS}

1. Cucinotta D; Vanelli M. WHO Declares COVID-19 a Pandemic [Internet]. Acta Biomed; 2020 Mar. [citado em 5 Jul 2020]; 91(1):157-160. Disponível em: https://doi.org/10.23750/abm.v91i1.9397.

2. Patanavanich R, Glantz SA. Smoking is Associated with COVID-19 Progression: A Meta-Analysis Nicotine Tob Res [Internet]. 2020 May. [citado em 2020 Jul 8]. Disponível em: https://doi.org/10.1101/2020.04.13.20063669.

3. Instituto Nacional de Câncer (INCA). Tabagismo e Coronavírus. 2020. Atualizado em 29 Mai 2020. [citado em 2020 Jul 10]. Disponível em: https://www.inca.gov.br/campanhas/dia-mundial-sem-tabaco/2020/tabagismo-e-coronavirus.

4. Silva ALO; Moreira J; Martins SR. COVID-19 e tabagismo: uma relação de risco [Internet]. Cad. Saúde Pública; 2020. [citado em 2020 Jul 14]; 36(5): 1-4. Disponível em https://doi:10.1590/0102-311X00072020.

5. Ministério da Saúde (BR). Secretaria de Vigilância em Saúde. Boletim Epidemiológico n 1 [Internet]. 2020 Jan. [citado em 2020 Jul 14]. Disponível em: https://portalarquivos2.saude.gov.br/images/pdf/2020/janeiro/23/Boletim_epidemiologico_ SVS_04.pdf.

6. Ministério da Saúde (BR). Orientação para Manejo de Pacientes com COVID-19. [Internet]. 2020 Jan. [citado em 2020 Jul 15]. Disponível em: https://portalarquivos.saude.gov.br/images/pdf/2020/June/18/Covid19-Orientac--o--esManejoPacientes.pdf.

7. Ministério da Saúde (BR). Secretaria de Vigilância em Saúde. Ficha de Investigação de SG Suspeito de Doença Pelo Coronavírus 2019 - COVID-19 (B34.2) [Internet]. 2020. [citado em 2020 Jul 02]. Disponível em: https://egestorab.saude.gov. br/image/?file=20200429_N_Fichae-SUSVE_5220668342419003136.pdf.

8. Ministério da Saúde (BR). Guia Política Nacional de Atenção Básica. Módulo 1: Integração Atenção Básica e Vigilância em Saúde. [citado em 2020 Jul 22]. Disponível em: http://bvsms.saude.gov.br/bvs/publicacoes/guia_politica_nacional_atencao_ basica_integracao_atencao_basica_vigilancia_saude_modulo_1.pdf.

9. Secretaria de Estado da Saúde (PR). Boletim Epidemiológico COVID-19, 29 de Julho de 2020 [Internet]. [citado em 2020 Jul 30]. Disponível em: http://www.saude.pr.gov.br/sites/default/arquivos_restritos/files/documento/2020-07/INFORME_ EPIDEMIOLOGICO_29_07_2020\%20.pdf.

10. WHO Framework Convention on Tobacco Control. Increased risk of COVID-19 infection amongst smokers and amongst waterpipe users. 2020. [citado em 2020 Jul 10]. Disponível em: http://www.emro.who.int/tfi/know-the-truth/tobacco-andwaterpipe-users-are-at-increased-risk-of-covid-19-infection.html. 
11. Mehra MR, Desai SS, Kuy S, Henry TD, Patel AN. Cardiovascular disease, drug therapy, and mortality in Covid-19 [Internet]. N Engl J Med; 2020. [citado em 2020 Jul 20]; 1-7. Disponível em: https://doi:10.1056/NEJMoa2007621.

12. Cen $Y$, Chen $X$, Shen $Y$, Zhang $X H$, Lei $Y, X u C$, et al. Risk factors for disease progression in patients with mild to moderate coronavirus disease 2019 - a multi-centre observational study [Internet]. Clin Microbiol Infect; 2020 June. [citado em 2020 Jul 21]. Disponível em: https://www.doi.org/10.1016/j.cmi.2020.05.041.

13. Zhao Q, Meng M, Kumar R, Wu Y, Huang J, Lian N, et al. The impact of COPD and smoking history on the severity of COVID-19: a systemic review and meta-analysis [Internet]. J Med Virol; 2020 Apr. [citado em 2020 Jul 21]; 1-6. Disponível em: https://doi.org/10.1002/jmv.25889.

14. Guan WJ, Liang WH, Zhao Y, Liang HR, Chen ZS, Li YM, et al. Comorbidity and its impact on 1590 patients with Covid-19 in China: a nationwide analysis [Internet]. Eur Respir J; 2020 Mar. [citado em 2020 Jul 21]; 56 (1). Disponível em: https://doi. org/10.1183/13993003.00547-2020.

RECEBIDO: 31/07/2020

ACEITO: $13 / 10 / 2020$ 